\title{
X-RAY VALIABILITIES FROM PROTOSTARS IN THE RCRA MOLECULAR CLOUD
}

KENJI HAMAGUCHI, HIROSHI MURAKAMI AND KATSUJI KOYAMA

Department of Physics, Faculty of Science, Kyoto University

Sakyo-ku, Kyoto 606-01, Japan

AND

SHIRO UENO

Department of Physics $\&$ Astronomy, University of leicester Leicester, LE1 7RH, U.K.

\section{Introduction}

$\mathrm{R}$ CrA molecular cloud, at a distance of 130pc(Marraco, Rydgren 1981), is one of the active low-to-middle mass star forming regions. The core of this cloud, named Coronet, contains five protostar candidates. In 1994, we observed the Coronet Cluster using the X-ray satellite ASCA, and discovered hard X-rays from protostar candidates(Koyama et al. 1996).

T-Tauri stars are known to exhibit the strong X-ray variablities with occasional rapid flares, whose emission mechanisms are similar to the solartype magnetic reconnection process(Montmerle et al. 1983), and the emitting regions are thought to be as large as the diameter of T-Tauri stars. On the other hand, the origin of protostar variabilities is less clear.

In 1996, we observed this region again, and found that the fluxes of each protostar candidates are different from those in 1994. This is the first evidence for the long time variablities of protostar candidates.

\section{Observations and Results}

The quesient state images for the $4-10 \mathrm{keV}$ band in 1994 and 1996 are shown in figure 1. The gray scale peaks will correspond to the position of IR cataloged protostar sources. One of the prominent feature is that, at the region centered on the protostar candidate, R1(R.A $=18^{h} 58^{m} 32.7^{s}$, DEC 


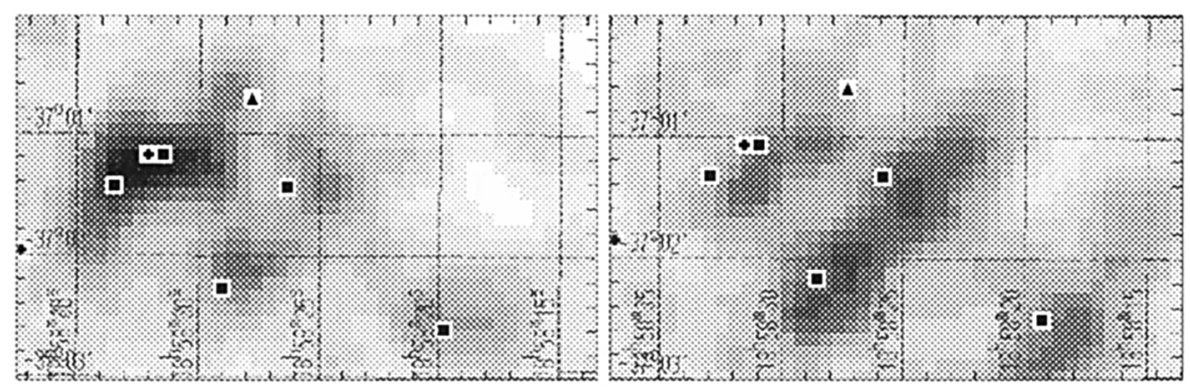

Figure 1. SIS(ASCA X-ray CCD Camera) images from the the Coronet cluster for the 4-10keV band in 1994(left side) and 1996(right side). Gray scales are normalized for the surface flux density. Squares are protostar candidates, crosses are Herbig $\mathrm{Ae} / \mathrm{Be}$ stars and a triangle is a classical T-Tauri star

$=-37^{\circ} 01^{\prime} 39^{\prime \prime}(1950)$, upper left on fig. 1 ), the flux in 1996 was at least 2.5 times lower than that in 1994. On the other hand, the fluxes of other sources are increased by $\sim 1.5$.

In the observation of 1994, we detected a powerful X-ray flare from one of the sources, probably the embedded far-infrared source R1. The X-ray flux increased at least by a factor of 3-4 from a queisent level. The flare spectrum is unusual, showing a broadend or double emission lines between 6.2 and $6.8 \mathrm{keV}$ (Koyama et al. 1996).

\section{Discussion}

What is the emission mechanism of these time valiabilities?

The flux reduction of R1 from 1994 to 1996 may be the feature like FU-Ori outbursts, which occur by the change of accretion rates.

The flare in 1994 is probably due to a solar-type magnetic activity like those seen in T-Tauri stars, but the flare spectrum is mysterious because there is no candidate line near $6.2 \mathrm{keV}$. One possibility is that these lines are doppler-shifted iron lines in a bipolar jet, whose speed is $\sim 0.1 \mathrm{c}$. However, no star forming theory predicts such a relativistic acceralation mechanism.

\section{References}

Marraco H G., Rydgren A.E. 1981 AJ 86, 62

Koyama K., Hamaguchi K., Ueno S., Kobayashi N., Eric D. Feigelson. 1996 PASJ 48, L87 Montmerle T., Koch-Miramond K., Falgarone E., Grindlay J.E. 1983, ApJ 269, 182 